International Journal of Pure and Applied Mathematics

Volume 83 No. 2 2013, 233-245

ISSN: 1311-8080 (printed version); ISSN: 1314-3395 (on-line version)

url: http://www.ijpam.eu

doi: http://dx.doi.org/10.12732/ijpam.v83i2.2

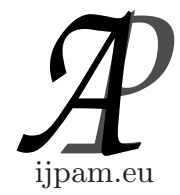

\title{
SEMIPRIME GAMMA RINGS WITH ORTHOGONAL REVERSE DERIVATIONS
}

\author{
Kalyan Kumar Dey ${ }^{1}$, Akhil Chandra Paul ${ }^{2}$, Isamiddin S. Rakhimov ${ }^{3} \S$ \\ ${ }^{1,2}$ Department of Mathematics \\ Rajshahi University \\ Rajshahi, 6205, BANGLADESH \\ ${ }^{3}$ Department of Mathematics \\ FS, \& Institute for Mathematical Research (INSPEM) \\ Universiti Putra Malaysia \\ MALAYSIA
}

\begin{abstract}
In this paper, the definition of orthogonal reverse derivations is given. Some characterizations of semiprime gamma rings are obtained by means of orthogonal reverse derivations. We also investigate conditions for two reverse derivations to be orthogonal.
\end{abstract}

AMS Subject Classification: 16A70, 16N60, 16W25

Key Words: semiprime gamma ring, derivation, reverse derivation, orthogonal reverse derivation

\section{Introduction}

In this section we review results on reverse derivations. The reverse derivations on semiprime rings have been studied by Samman and Alyamani [9]. Here the authors obtain some characterizations of semiprime rings by the help of reverse derivations. Sapanci and Nakajima [9] investigated the commutativity properties of a gamma ring with the derivations. Bresar and Vukman [6]

Received: June 25, 2012

(C) 2013 Academic Publications, Ltd.

$\S$ Correspondence author url: www.acadpubl.eu 
initiated the notion of orthogonality for two derivations on a semiprime ring. Some necessary and sufficient conditions for two derivations to be orthogonal are obtained. They also obtained a counterpart of a result of Posner from [8]. Argac, Nakajima and Albas [1] worked on orthogonal generalized derivations on a semiprime ring and they established some results concerning two generalized derivations on a semiprime ring. Ozturk, Jun and Kim [7] worked on prime $\Gamma$-rings by means of derivations.

In this paper, we extend the results mentioned above to gamma rings case. The notion of orthogonality of two reverse derivations is given and conditions of two reverse derivations to be orthogonal are provided. We also obtain some characterizations of a semiprime gamma rings with orthogonal reverse derivations.

\section{Premilinaries}

Let $M$ and $\Gamma$ be additive abelian groups. If there exists a mapping $M \times \Gamma \times M \rightarrow$ $M:(x, \alpha, y) \rightarrow x \alpha y$ which satisfies the conditions: for all $a, b, c \in M, \alpha, \beta \in \Gamma$,

1. $(a+b) \alpha c=a \alpha c+b \alpha c, a(\alpha+\beta) b=a \alpha b+a \beta b, a \alpha(b+c)=a \alpha b+a \alpha c$,

2. $(a \alpha b) \beta c=a \alpha(b \beta c)$,

then $M$ is called a $\Gamma$-ring.

The concepts of subring and ideal are imitated from the classical case. Throughout the paper, $M$ denotes a $\Gamma$-ring with center $Z(M)$. A ring $M$ is said to be 2 -torsion free if $2 x=0$ for $x \in M$ implies $x=0$. We write $[x, y]_{\alpha}$ for $x \alpha y-y \alpha x$. Recall that a $\Gamma$-ring $M$ is called prime if $a \Gamma M \Gamma b=0$ implies $a=$ 0 or $b=0$, and it is called semiprime if $a \Gamma M \Gamma a=0$ implies $a=0$. A prime $\Gamma$-ring is obviously semiprime. A $\Gamma$-ring $M$ is called commutative if $[x, y]_{\alpha}=0$ for every $x, y \in M$ and $\alpha \in \Gamma$. An additive mapping $d$ from $M$ into itself is called a derivation if $d(x \alpha y)=d(x) \alpha y+x \alpha d(y)$, for all $x, y \in M, \alpha \in \Gamma$. We consider an assumption (*) $x \alpha y \beta z=x \beta y \alpha z$ for all $x, y, z \in M, \alpha, \beta \in \Gamma$. The basic commutator identities given by $[x \beta y, z]_{\alpha}=x \beta[y, z]_{\alpha}+[x, z]_{\alpha} \beta y+x[\beta, \alpha]_{z} y$ and $[x, y \beta z]_{\alpha}=y \beta[x, z]_{\alpha}+[x, y]_{\alpha} \beta z+y[\beta, \alpha]_{\mathrm{x}} z$, for all $x, y, z \in M$ and for all $\alpha, \beta \in \Gamma$. Taking the above assumption $(*)$ the basic commutator identities reduce to $[x \beta y, z]_{\alpha}=x \beta[y, z]_{\alpha}+[x, z]_{\alpha} \beta y$ and $[x, y \beta z]_{\alpha}=y \beta[x, z]_{\alpha}+[x$, $y]_{\alpha} \beta z$, for all $x, y, z \in M$ and for all $\alpha, \beta \in \Gamma$ which are used extensively in our results. 


\section{Reverse Derivation and Orthogonal Reverse Derivation}

An additive mapping $d$ from a $\Gamma$-ring $M$ into itself satisfying $d(x \alpha y)=d(y) \alpha x+$ $\operatorname{yod}(x)$, for all $x, y \in M, \alpha \in \Gamma$, is called a reverse derivation. Obviously, if $M$ is commutative, then both derivation and reverse derivation are the same. An additive mapping $d: M \rightarrow M$ is called a Jordan derivation if $d(a \alpha a)=$ $d(a) \alpha a+\operatorname{a\alpha d}(a)$ for all $a \in M$ and $\alpha \in \Gamma$. It can be easily seen that the reverse derivation is not a derivation in general, but it is a Jordan derivation.

Example 3.1. Let $R$ be an associative ring with $1, d: R \rightarrow R$ be a reverse derivation. Consider $M=M_{1,2}(R)$ and $\Gamma=\left\{\left(\begin{array}{l}n \cdot 1 \\ 0\end{array}\right): n \in \mathrm{Z}\right\}$. Then it is clear that $M$ is a $\Gamma$-ring. Let $N=\{(x, x): x \in R\} \subset M$.

Then $N$ is a subring of $M$. Define $D: N \rightarrow N$ by $D((x, x))=(d(x), d(x))$. If $\mathrm{a}=\left(x_{1}, x_{1}\right), \mathrm{b}=\left(x_{2}, x_{2}\right)$ and $\alpha=\left(\begin{array}{l}n \cdot 1 \\ 0\end{array}\right) \in \Gamma$. Then we have

$$
\begin{aligned}
D(a \alpha b) & =D\left(\left(x_{1}, x_{1}\right)\left(\begin{array}{l}
n \cdot 1 \\
0
\end{array}\right)\left(x_{2}, x_{2}\right)\right) \\
& =D\left(x_{1} n x_{2}, x_{1} n x_{2}\right) \\
& =\left(d\left(x_{1} n x_{2}\right), d\left(x_{1} n x_{2}\right)\right) \\
& =\left(d\left(x_{2}\right) n x_{1}+x_{2} n d\left(x_{1}\right), d\left(x_{2}\right) n x_{1}+x_{2} n d\left(x_{1}\right)\right) \\
& =\left(d\left(x_{2}\right) n x_{1}, d\left(x_{2}\right) n x_{1}\right)+\left(x_{2} n d\left(x_{1}\right), x_{2} n d\left(x_{1}\right)\right) \\
& =\left(d\left(x_{2}\right), d\left(x_{2}\right)\right)\left(\begin{array}{l}
n \cdot 1 \\
0
\end{array}\right)\left(x_{1}, x_{1}\right)+\left(x_{2}, x_{2}\right)\left(\begin{array}{l}
n \cdot 1 \\
0
\end{array}\right)\left(d\left(x_{1}\right), d\left(x_{1}\right)\right) \\
& =D\left(\left(x_{2}, x_{2}\right)\right) \alpha a+b \alpha D\left(\left(x_{1}, x_{1}\right)\right) \\
& =D(b) \alpha a+b \alpha D(a) .
\end{aligned}
$$

Hence $D$ is a reverse derivation on $\Gamma$-ring $N$.

Now we give the definition of orthogonality of two reverse derivations.

Definition 3.2. Let $d$ and $g$ be two reverse derivations on $M$. If

$$
d(x) \Gamma M \Gamma g(y)=0=g(y) \Gamma M \Gamma d(x) \text { for all } x, y \in M .
$$

Then $d$ and $g$ are said to be orthogonal.

Note that a non-zero reverse derivation can not be orthogonal on itself.

Example 3.3. Let $M_{1}$ be a $\Gamma_{1}$-ring and let $M_{2}$ be a $\Gamma_{2}$-ring. Consider $M=M_{1} \times M_{2}$ and $\Gamma=\Gamma_{1} \times \Gamma_{2}$. The addition and multiplication on $M$ and 
$\Gamma$ are defined as follows:

$$
\begin{gathered}
(a, b)+(c, d)=(a+c, b+d), \\
(a, b)(\alpha, \beta)(c, d)=(a \alpha c, b \beta d) \text { foreverya, } b \in M_{1}, \\
c, d \in M_{2}, \quad \alpha \in \Gamma_{1} \text { and } \beta \in \Gamma_{2} .
\end{gathered}
$$

Under these operations $M$ is a $\Gamma$-ring. Let $d_{1}$ be a reverse derivation on $M_{1}$. Define a derivation $d$ on $M$ by $d((a, b))=\left(d_{1}(a), 0\right)$. Then $d$ is a reverse derivation on $M$. Let $d_{2}$ be a reverse derivation on $M_{2}$. Define a derivation $g$ on $M$ by $\mathrm{g}((a, b))=\left(0, d_{2}(b)\right)$. Then $g$ is a reverse derivation on $M$. It is clear that $\mathrm{d}$ and $\mathrm{g}$ are orthogonal reverse derivation on $\mathrm{M}$.

\section{Results}

We start this section by some observations which are useful in proving our main results. The following result has been given in [2],

Lemma 4.1. Let $M$ be a 2-torsion free semiprime $\Gamma$-ring and $a, b \in M$. Then the following conditions are equivalent:

1. $a \Gamma x \Gamma b=0$, for all $x \in M$.

2. $b \Gamma x \Gamma a=0$, for all $x \in M$.

(iii) $a \Gamma x \Gamma b+b \Gamma x \Gamma a=0$, for all $x \in M$.

If one of these conditions is fulfilled then $a \Gamma b=0=b \Gamma a$.

Lemma 4.2. Let $M$ be a semiprime $\Gamma$-ring and suppose that additive mappings $d$ and $g$ of $M$ into itself satisfy $d(x) \Gamma M \Gamma g(x)=0$, for all $x \in M$. Then $d(x) \Gamma M \Gamma g(y)=0$, for all $x \in M$.

Proof. Suppose that $d(x) \alpha m \beta g(x)=0$, for all $x, m \in M, \alpha, \beta \in \Gamma$. Replace $x$ by $x+y$ in the above relation, we get

$$
\begin{aligned}
0 & =d(x+y) \alpha m \beta g(x+y)=(d(x)+d(y)) \alpha m \beta(g(x)+g(y)) \\
& =d(x) \alpha m \beta g(x)+d(x) \alpha m \beta g(y)+d(y) \alpha m \beta g(x)+d(y) \alpha m \beta g(y) \\
& =d(x) \alpha m \beta g(y)+d(y) \alpha m \beta g(x) . T h u s d(x) \alpha m \beta g(y)=-d(y) \alpha m \beta g(x) .
\end{aligned}
$$

Now

$$
(d(x) \alpha m \beta g(y)) \gamma n \delta(d(x) \alpha m \beta g(y))=(d(x) \alpha m \beta g(y)) \gamma n \delta(-d(y) \alpha m \beta g(x))
$$




$$
=-(d(x) \alpha m \beta g(y) \gamma n \delta d(y) \alpha m \beta g(x)=0,
$$

for all $x, y, m, n \in M, \alpha, \beta, \delta \in \Gamma$.

Thus $d(x) \Gamma M \Gamma g(y)=0$, for all $x, y \in M$.

Lemma 4.3. Let $M$ be a 2-torsion free semiprime $\Gamma$-ring. Let $d$ and $g$ be reverse derivations of $M$. Then

$$
d(x) \Gamma g(y)+g(x) \Gamma d(y)=0, \quad \text { for all } x, y \in M .
$$

if and only if $d$ and $g$ are orthogonal.

Proof. Suppose that $d(x) \alpha g(y)+g(x) \alpha d(y)=0$, for all $x, y \in M, \alpha \in \Gamma$.

Consider the substitution $y=x \beta y$ in (2). Then we obtain

$$
\begin{aligned}
& 0=d(x) \alpha g(x \beta y)+g(x) \alpha d(x \beta y), \\
& 0=d(x) \alpha(g(y) \beta x+y \beta g(x))+g(x) \alpha(d(y) \beta x+y \beta d(x), \\
& 0=(d(x) \alpha g(y)+g(x) \alpha d(y)) \beta x+d(x) \alpha y \beta g(x)+g(x) \alpha y \beta d(x) .
\end{aligned}
$$

Using (2), we have $d(x) \alpha y \beta g(x)+g(x) \alpha y \beta d(x)=0$. Then due to Lemma 4.2 , we get $d(x) \alpha y \beta g(x)=0$, which gives the orthogonality of $d$ and $g$.

Conversely, if $d$ and $g$ are orthogonal, we get $d(x) \alpha m \beta g(y)=g(x) \alpha m \beta d(y)=$ 0 for all $m \in M, \alpha, \beta \in \Gamma$. Then by using Lemma 4.1, we obtain $d(x) \alpha g(y)=$ $g(x) \alpha d(y)=0$, for all $x, y \in M, \alpha \in \Gamma$. Thus $d(x) \alpha g(y)+g(x) \alpha d(y)=0$, for all $x, y \in M, \alpha \in \Gamma$ which completes the proof.

Suppose that $d$ and $g$ are reverse derivations of a $\Gamma$-ring $M$. The following identities are immediate from the definition of reverse derivation.

$$
\begin{array}{r}
(d g)(x \alpha y)=d(g(x \alpha y))=d(g(y) \alpha x+y \alpha g(x))=(d g)(x) \alpha y+d(x) \alpha g(y) \\
+g(x) \alpha d(y)+x \alpha(d g)(y) \text { for all } x, y \in M, \alpha \in \Gamma .
\end{array}
$$

Similarly,

$$
\begin{array}{r}
(g d)(x \alpha y)=g(d(x \alpha y))=g(d(y) \alpha x+y \alpha d(x))=(g d)(x) \alpha y+g(x) \alpha d(y) \\
+d(x) \alpha g(y)+x \alpha(g d)(y) \text { for all } x, y \in M, \alpha \in \Gamma .
\end{array}
$$

The following theorem gives a few criteria on orthogonality of reverse derivations. It is an extension of Theorem 2.1 from [2] to the reverse derivations case.

Theorem 4.4. Let $M$ be a 2-torsion free semiprime $\Gamma$-ring. Let $d$ and $g$ be reverse derivations on $M$. Then the following conditions are equivalent: 
(i) $d$ and $g$ are orthogonal.

(ii) $d g=0$.

(iii) $g d=0$.

(iv) $d g+g d=0$.

(v) $d g$ is a derivation.

(vi) $g d$ is a derivation.

Proof. (ii) $\Rightarrow(\mathrm{i})$. Suppose $d g=0$. Then by using the identity (3) above we obtain

$$
d(x) \alpha g(y)+g(x) \alpha d(y)=0, \text { for all } x, y \in M, \alpha \in \Gamma \text {. }
$$

Therefore by Lemma $4.3, d$ and $g$ are orthogonal

1. $\Rightarrow$ (ii). Consider $d(x) \alpha y \beta g(z)=0$, for all $x, y, z \in M, \alpha, \beta \in \Gamma$.

Then

$$
\begin{aligned}
0=d(d(x) \alpha y \beta g(z)) & =d(y \beta g(z)) \alpha d(x)+y \beta g(z) \alpha d^{2}(x) \\
& =(d g)(z) \beta y \alpha d(x)+g(z) \beta d(y) \alpha d(x)+y \beta g(z) \alpha d(d(x))
\end{aligned}
$$

Owing to (i), the second and third summands are zero. Therefore we obtain $(d g)(z) \beta y \alpha d(x)=0$ for all $x, y, z \in M, \alpha, \beta \in \Gamma$. Now take $x=g(z)$ and we obtain

$$
(d g)(z) \beta y \alpha(d g)(z)=0 \text {, for all } z \in M, \alpha, \beta \in \Gamma \text {. }
$$

Since $M$ is semiprime, we get $(d g)(z)=0$, for all $z \in M$., that is $d g=0$.

The proof of the parts (iii) $\Rightarrow$ (i) and (i) $\Rightarrow$ (iii) are similar.

(iv) $\Rightarrow$ (i). If $d$ and $g$ are any reverse derivations, then by (ii) and (iii), $d g$ $=0$ and $g d=0$.

Now using the equation (3), we obtain,

$$
\begin{aligned}
(d g+g d)(x \alpha y)= & (d g)(x \alpha y)+(g d)(x \alpha y) \\
= & (d g)(x) \alpha y+d(x) \alpha g(y)+g(x) \alpha d(y)+x \alpha(d g)(y)+(g d)(x) \alpha y \\
& +g(x) \alpha d(x)+d(x) \alpha g(y)+x \alpha(g d)(y) \\
= & (d g+g d)(x) \alpha y+2 d(x) \alpha g(y)+2 g(x) \alpha d(y) \\
& +x \alpha((d g)(y)+(g d)(y))
\end{aligned}
$$

for all $x, y \in M, \alpha \in \Gamma$. 
Thus, if $d g+g d=0$, then the above relation reduces to $2(d(x) \alpha g(y)+$ $g(x) \alpha d(y))=0$, for all $x, y \in M, \alpha \in \Gamma$. Since $M$ is 2-torsion free, we get

$$
d(x) \alpha g(y)+g(x) \alpha d(y)=0, \text { for all } x, y \in M, \alpha \in \Gamma .
$$

By Lemma 4.3, we get that $d$ and $g$ are orthogonal.

(i) $\Rightarrow$ (iv). From the parts (ii) and (iii) of Theorem 4.1, we get $d g+g d=$ 0 .

(v) $\Rightarrow$ (i). Since $d g$ is a derivation, we have

$$
(d g)(x \alpha y)=(d g)(x) \alpha y+x \alpha(d g)(y) .
$$

Comparing this expression with (3) we obtain

$$
d(x) \alpha g(y)+g(x) \alpha d(y)=0 .
$$

The proof of (vi) $\Rightarrow$ (i) is the similar to that of (v) $\Rightarrow$ (i).

(iii) $\Rightarrow$ (vi). Obvious.

This completes the proof.

Corollary 4.5. Let $M$ be a prime 2-torsion free $\Gamma$-ring. Suppose that $d$ and $g$ are orthogonal reverse derivations of $M$. Then either $d=0$ or $g=0$.

The proof is immediate from Theorem 4.4.

Theorem 4.6. Let $M$ be a 2-torsion free, semiprime $\Gamma$-ring satisfying the condition

$x \alpha y \beta z=x \beta y \alpha z$ for all $x, y, z \in M$ and $\alpha, \beta \in \Gamma$.

Let $d$ and $g$ be reverse derivations on $M$. Then the following conditions are equivalent:

(i) $d$ and $g$ are orthogonal.

(ii) $d(x) \Gamma g(x)=0$, for all $x \in M$.

(iii) $g(x) \Gamma d(x)=0$, for all $x \in M$.

(iv) $d(x) \Gamma g(x)+g(x) \Gamma d(x)=0$, for all $x \in M$.

Proof. (ii) $\Rightarrow$ (i) The linearization of $d(x+y) \alpha g(x+y)=0$ gives

$$
d(x) \alpha g(y)+d(y) \alpha g(x)=0, \text { for all } x, y \in M, \alpha \in \Gamma .
$$

Take $y \beta z$ as $y$ in (5), we obtain

$$
d(x) \alpha g(y \beta z)+d(y \beta z) \alpha g(x)=0, \text { for all } x, y, z \in M, \alpha, \beta \in \Gamma .
$$




$$
\begin{aligned}
d(x) \alpha g(z) \beta y+d(x) \alpha z \beta g(y)+d(z) \beta y \alpha g(x)+ & z \beta d(y) \alpha g(x)=0, \\
& \text { for all } x, y, z \in \Gamma, \alpha, \beta \in \Gamma .
\end{aligned}
$$

Since

$$
d(x) \alpha g(z)=-d(z) \alpha g(x) \text { and } d(y) \alpha g(x)=-d(x) \alpha g(y)
$$

and so the above relation becomes

$$
-d(z) \alpha g(x) \beta y+d(x) \alpha z \beta g(y)+d(z) \beta y \alpha g(x)-z \beta d(x) \alpha g(y)=0,
$$

for all $x, y, z \in M, \alpha, \beta \in \Gamma$.

Now we make use the condition $(*)$ then

$$
d(z) \beta[y, g(x)]_{\alpha}+[d(x), z]_{\alpha} \beta g(y)=0 .
$$

Replacing $z$ by $d(x)$ in the above identity we obtain

$$
d^{2}(x) \beta[y, g(x)]_{\alpha}=0
$$

for all $x, y \in M, \alpha, \beta \in \Gamma$.

Letting $y=y \delta w$ in the last relation and using the condition $\left(^{*}\right)$, we get,

$$
\begin{aligned}
0=d^{2}(x) \beta[y \delta w, g(x)]_{\alpha}=d^{2}(x) \beta y \delta[w, g(x)]_{\alpha}+d^{2}(x) \beta y \delta[w, g(x) & ]_{\alpha} \\
& =d^{2}(x) \beta y \delta[w, g(x)]_{\alpha}
\end{aligned}
$$

for all $x, y, w \in M, \alpha, \beta, \delta \in \Gamma$.

Then by using Lemma 4.2 , we obtain

$$
d^{2}(x) \beta y \delta[w, g(y)]_{\alpha}=0, \text { for all } x, y, w \in M, \alpha, \beta, \delta \in \Gamma .
$$

Replacing $x$ by $x \lambda u$ in (6) and using (3) yields

$$
0=d^{2}(x \lambda u) \beta y \delta[w, g(y)]_{\alpha}=\left(d^{2}(x) \lambda u+2 d(x) \lambda d(u)+x \lambda d^{2}(u)\right) \beta y \delta[w, g(y)]_{\alpha}
$$

for all $x \in M, \alpha, \beta, \delta, \lambda \in \Gamma$.

By (6) the above relation reduces to

$$
2 d(x) \lambda d(u) \beta y \delta[w, g(y)]_{\alpha}=0 .
$$

Since $M$ is 2 -torsion free, we have

$$
d(x) \lambda d(u) \beta y \delta[w, g(y)]_{\alpha}=0, \text { for all } x, y \in M, \alpha, \beta, \delta, \lambda \in \Gamma \text {. }
$$


It is obvious from the definition of $K$ that $d$ leaves $K$

Taking $x \gamma z$ for $x$ in (7), we get

$$
\begin{aligned}
0=d(x \gamma z) \lambda d(u) \beta y \delta[w, g(y)]_{\alpha} \\
\quad=d(z) \gamma x \lambda d(u) \beta y \delta[w, g(y)]_{\alpha}+z \gamma d(x) \lambda d(u) \beta z \delta[w, g(y)]_{\alpha}
\end{aligned}
$$

and by using (7), we get

$$
d(z) \gamma x \lambda d(u) \beta y \delta[w, g(y)]_{\alpha}=0 .
$$

In particular,

$$
d(z) \gamma x \lambda d(x) \beta y \delta[w, g(y)]_{\alpha}=0 .
$$

The replacement $d(z)=d(x) \beta y \delta[w, g(y)]_{\alpha}$, gives

$$
d(x) \beta y \delta[w, g(y)]_{\alpha} \gamma x \lambda d(x) \beta y \delta[w, g(y)]_{\alpha}=0 .
$$

Since $M$ is semiprime, we get $d(x) \beta y \delta[w, g(y)]_{\alpha}=0$.

Using (6) and (7) we obtain by replacing $d(x)$ for $w$,

$$
[d(x), g(y)]_{\alpha} \gamma y \delta[d(x), g(y)]_{\alpha}=0,
$$

for all $x, y \in M, \alpha, \beta, \delta, \gamma \in \Gamma$.

Hence

$$
d(x) \alpha g(y)=g(y) \alpha d(x), \text { for all } x, y \in M, \alpha \in \Gamma .
$$

Thus (5) can be written in the form

$$
g(y) \alpha d(x)+d(y) \alpha g(x)=0, \text { for all } x, y \in M, \alpha \in \Gamma .
$$

Now use Lemma 4.3 to get the required relation.

(i) $\Rightarrow$ (iii). If $d$ and $g$ are orthogonal then we have

$$
d(x) \Gamma M \Gamma g(x)=0, \text { for all } x \in M .
$$

Then due to Lemma 4.1, we get

$$
d(x) \alpha g(x)=0, \text { for all } x \in M, \alpha \in \Gamma .
$$

(iii) $\Rightarrow$ (ii). Take $y=x$ in (3). Then we see that

$$
(d g)(x \alpha x)=(d g)(x) \alpha x+d(x) \alpha g(x)+g(x) \alpha d(x)+x \alpha(d g)(x) .
$$


Thus we obtain

$$
(d g)(x \alpha x)=(d g)(x) \alpha x+x \alpha(d g)(x), \text { for all } x \in M, \alpha \in \Gamma \text {. }
$$

The above relation implies that $d g$ is a Jordan derivation. We know that if $M$ is semiprime $\Gamma$-ring, then every Jordan derivation is a derivation.

(i) $\Rightarrow$ (ii). This follows immediately from Lemma 4.3 .

Corollary 4.7. Let $M$ be a 2-torsion free semiprime $\Gamma$-ring and let $d$ be a reverse derivation of $M$. If $d^{2}$ is also a derivation, then $d=0$.

The proof follows from part (ii) of Theorem 4.6.

Theorem 4.8. Let $M$ be a 2-torsion free semiprime $\Gamma$-ring. Let $d$ and $g$ be reverse derivations on $M$. Then the following conditions are equivalent:

(i) $d$ and $g$ are orthogonal.

(ii) There exist ideals $K_{1}$ and $K_{2}$ of $M$ such that:

(a) $K_{1} \cap K_{2}=0$ and $K=K_{1} \oplus K_{2}$ is a nonzero ideal of $M$.

(b) $d$ maps $M$ into $K_{1}$ and $g$ maps $M$ into $K_{2}$.

(c) The restriction of $d$ to $K=K_{1} \oplus K_{2}$ is a direct sum $d_{1} \oplus 0_{2}$, where $d_{1}$ : $K_{1} \rightarrow K_{1}$ is a reverse derivation of $K_{1}$ and $0_{2}: K_{2} \rightarrow K_{2}$ is zero. If $d_{1}=0$ then $d=0$.

(d) The restriction of $g$ to $K=K_{1} \oplus K_{2}$ is a direct sum $0_{1} \oplus g_{2}$, where $0_{1}$ : $K_{1} \rightarrow K_{1}$ is zero and $g_{2}: K_{2} \rightarrow K_{2}$ is a reverse derivation of $K_{2}$. If $g_{2}=0$ then $g=0$.

Proof. (ii) $\Rightarrow$ (i). Obvious.

(i) $\Rightarrow$ (ii). Let $K_{1}$ be an ideal of $M$ generated by all $d(x), x \in M$, and let $K_{2}$ be $A n n\left(K_{1}\right)$, the annihilator of $K_{1}$. From (1) we see that $g(x) \in K_{2}$, for all $x \in M$. Whenever $K_{1}$ is an ideal in a semiprime $\Gamma$-ring, we have $K_{1} \cap K_{2}=0$ and $K=K_{1} \oplus K_{2}$ is a nonzero ideal. Thus (a) and (b) are proved.

Our next goal is to show that $d$ is zero on $K_{2}$. Take $k_{2} \in K_{2}$. Then $k_{1} \alpha k_{2}=0$, for all $k_{1} \in K_{1}, \alpha \in \Gamma$. Hence $0=d\left(k_{1} \alpha k_{2}\right)=d\left(k_{2}\right) \alpha k_{1}+k_{2} \alpha d\left(k_{1}\right)$. It is obvious from the definition of $K$ that $d$ leaves $K_{1}$ invariant and hence $k_{2} \alpha d\left(k_{1}\right)=0$. Then the above relation reduces to $d\left(k_{2}\right) \alpha k_{1}=0$. Since in a semiprime $\Gamma$-ring the left, right and two-sided annihilators of an ideal coincide, we then have $d\left(k_{2}\right) \in A n n\left(K_{1}\right)=K_{2}$. But on the other hand $d\left(k_{2}\right)$ belongs to the set of generating elements of $K_{1}$. Thus $d\left(k_{2}\right) \in K_{1} \cap K_{2}=0$, which means that $d$ is zero on $K_{2}$. As we have mentioned above $d$ leaves $K_{1}$ invariant. Therefore we may define a mapping $d_{1}: K_{1} \rightarrow K_{1}$ as a restriction of $d$ to $K_{1}$. 
Suppose that $d_{1}=0$. Then $d$ is zero on $K=K_{1} \oplus K_{2}$. Take $k \in K$ and $y \in M$, we have

$d(y \alpha k)=d(k) \alpha y+k \alpha d(y)$

But $d(y \alpha k)=d(k)=0$ since $k \alpha y, k \in K, \alpha \in \Gamma$. Consequently $k \alpha d(y)=$ 0 , for all $y \in M, \alpha \in \Gamma$. Thus $d(y) \in A n n(K)$. But ideal $K$ is nonzero and therefore $\operatorname{Ann}(K)=0$. Hence $d(y)=0$, for all $y \in M$.

Then (c) is thereby proved.

It remains to prove (d). First we show that $g$ is zero on $K_{1}$. Take $x, y$, $z \in M, \alpha, \beta \in \Gamma$ and set $k_{1}=z \alpha d(y) \beta x$. Then

$$
\begin{aligned}
g\left(k_{1}\right)=g(x) \beta(z \alpha d(y))+x \beta & g(z \alpha d(y)) \\
& =g(x) \beta z \alpha d(y)+x \beta(g d)(y) \alpha z+x \beta d(y) \alpha g(z) .
\end{aligned}
$$

Since $d$ and $g$ are orthogonal we have $g(x) \alpha z \beta d(y)=0, d(y) \alpha g(z)=0$ and $g d=0$. Hence $g\left(k_{1}\right)=0$. In a similar fashion we see that $g(z \alpha d(y))=0$, $g(d(y) \alpha x)=0$ and $g(d(y))=0$. Then $h$ is zero on $K_{1}$. Recall that $g$ maps $M$ into $K_{2}$. In particular, it leaves $K_{2}$ invariant. Thus we may define

$g_{2}: K_{2} \rightarrow K_{2}$ as a restriction of $g$ to $K_{2}$. The proof that $g_{2}=0$ implies $g=$ 0 is the same as the proof that $d_{1}=0$ implies $d=0$. This completes the proof.

Corollary 4.9. Let $M$ be a 2-torsion free semiprime $\Gamma$-ring and let $d$ be a reverse derivation of $M$. If $d(x) \alpha d(x)=0$ for all $x \in M, \alpha \in \Gamma$, then $d=0$.

If $d^{2}=g^{2}$ or if $d(x) \alpha d(x)=g(x) \alpha g(x)$, for every $x \in M, \alpha \in \Gamma$, then we obtain the relation between the reverse derivations $d$ and $g$ of a $\Gamma$-ring.

Theorem 4.10. Let $M$ be a 2-torsion free semiprime $\Gamma$-ring. Let $d$ and $g$ be reverse derivations of $M$. Suppose that $d^{2}=g^{2}$, then $d+g$ and $d-g$ are orthogonal. Thus, there exist ideals $K_{1}$ and $K_{2}$ of $M$ such that $K=K_{1} \oplus K_{2}$ is a nonzero ideal which is direct sum in $M, d=g$ on $K_{1}$ and $d=-g$ on $K_{2}$.

Proof. From $d^{2}=g^{2}$ it follows immediately that $(d+g)(d-g)+(d-$ $g)(d+g)=0$. Hence $d+g$ and $d-g$ are orthogonal by the part (iii) of Theorem 4.4. Another part of Theorem 4.10, follows from (iii) of Theorem 4.8.

From Theorem 4.10 we get the following

Corollary 4.11. Let $M$ be a prime 2-tosion free $\Gamma$-ring. Let $d$ and $g$ be derivations of $M$. If $d^{2}=g^{2}$ then either $d=-g$ or $d=g$.

Theorem 4.12. Let $M$ be a 2-torsion free semiprime $\Gamma$-ring. Let $d$ and $g$ be reverse derivations of $M$. If $d(x) \alpha d(x)=g(x) \alpha g(x)$, for all $x \in M, \alpha \in \Gamma$, then $d+g$ and $d-g$ are orthogonal. Thus, there exist ideals $K_{1}$ and $K_{2}$ of $M$ 
such that $K=K_{1} \oplus K_{2}$ is an essential direct sum in $M, d=g$ on $K_{1}$ and $d=$ $-g$ on $K_{2}$.

Proof. Note that $(d+g)(x) \alpha(d-g)(x)+(d-g)(x) \alpha(d+g)(x)=0$, for all $x \in M, \alpha \in \Gamma$. Now applying parts (ii) and (iii) of Theorem 4.6, we obtain the required result.

Corollary 4.13. Let $M$ be a prime 2 -torsion free $\Gamma$-ring. Let $d$ and $g$ be reverse derivations of $M$. If $d(x) \alpha d(x)=g(x) \alpha g(x)$, for all $x \in M, \alpha \in \Gamma$, then either $d=g$ or $d=-g$.

The proof is immediate from Theorem 4.12.

\section{Acknowledgments}

The first named author thanks the Institute for Mathematical Research (INSPEM), UPM, Malaysia for the hospitality during that this paper was written. The research was supported by grant 01-12-10-978FR MOHE, Malaysia.

\section{References}

[1] N. Argac, A. Nakajima, E. Albaş, On orthogonal generalized derivations of semiprime rings, Turk. J. Math., 28 (2004), 185-194.

[2] M. Ashraf, M.R. Jamal, Orthogonal derivations in $\Gamma$-rings, Advances in Algebra, 3, No. 1 (2010), 1-6.

[3] W.E. Barnes, On the $\Gamma$-rings of Nabusawa, Pacific J. Math., 18 (1966), 411-422.

[4] M. Bresar, Jordan derivations on semi-prime rings, Proc. Amer. Math. Soc., 104 (1988), 1003-1006.

[5] M. Bresar, J. Vukman, On some additive mappings in rings with involution, Aequationes Mathematicae., 38 (1989), 178-185.

[6] M. Bresar, J. Vukman, Orthogonal derivations and an extension of a theorem of Posner, Radovi Matematicki, 5 (1991), 237-246.

[7] M.A. Ozturk, Y.B. Jun, K.H. Kim, On derivations of prime gamma rings, Turk. J. Math., 26 (2002), 317-327. 
[8] E. Posner, Derivations in prime rings, Proc. Amer. Math. Soc., 8 (1957), 1093-1100.

[9] M. Samman, N. Alyamani, Derivations and reverse derivations in semiprime rings, International Mathematical Forum, 2, No. 39 (2007), 1895-1902.

[10] M. Sapanci, A. Nakajima, A note on gamma rings, Turk. J. Math., 20 (1996) 463-465.

[11] N.N. Sulaiman, A.R.H. Majeed, Orthogonal derivations on an ideal of semiprime $\Gamma$-rings, International Mathematical Forum, 7, No. 28 (2012), 1405-1412. 
